\title{
Correlation between Craniofacial Structures and Severity of Obstructive Sleep Apnea
}

Domenico Ciavarella, Michele Laurenziello*, Lucio Lo Russo, Matteo Vocale, Michele Tepedino, Ferruccio Madaro, Lorenzo Lo Muzio and Michele Cassano

*Department of Clinical and Experimental Medicine, University of Foggia, Foggia, Italy

*Corresponding author: Dr. Michele Laurenziello, Department of Clinical and Experimental Medicine, University of Foggia, Foggia, Italy, Tel: 3890287888; E-mail: dr.mlaurenziello@gmail.com

Received date: December 18, 2018; Accepted date: December 24, 2018; Published date: December 31, 2018

Copyright: (c) 2018 Ciavarella D, et al. This is an open-access article distributed under the terms of the Creative Commons Attribution License, which permits unrestricted use, distribution, and reproduction in any medium, provided the original author and source are credited.

\begin{abstract}
Objective: In the present paper authors evaluated, on supine lateral head films, the correlation of Upper Airway Space (UAS), posterior maxillary structures (i.e., palatal morphology), maxillo-mandibular and hyoid position with different severity of OSA evaluated on polysomnography test.

Materials and Methods: One hundred patients (mean age 51.4 years old; 92 man and 8 women) with OSA were en-rolled in the present study. An Epworth questionnaire and a complete overnight Polysomnography (PSG) were done. Head films were taken with the patient in supine position fixed in a cephalostat, in centric occlusion, with adequate visualization of reference structures.
\end{abstract}

Results: The cephalometric evaluation showed how patients presented a reduction of Upper airway space, a post inclination of the mandible, an increase of the ANB Angle and an increase of palatal length, palatal height and palatal angle. At Spearman test a great correlation between IAS and OSA data, a direct correlation between the palatal length and thickness with IAS and an inverse correlation between the mandibular post inclination and backward position and IAS were evaluated.

Conclusion: In the present paper authors showed how PSG (AHI, SO2 and Nadir) data was correlated above all to IAS. The modification of IAS was related to the mandibular vertical and sagittal position and to palatal length and thickness.

Keywords: OSA; Polysomnography; Cephalometric analysis; AHI; Upper airway space

\section{Abbreviation \\ OSAs: Obstructive Sleep Apnea syndrome; ESS: Epworth Sleepiness Scale; UAS: Upper Airway Space; BMI: Body Mass Index; CPAP: Continuous Positive Airway Pressure; nCPAP: non-Continuous Positive Airway Pressure; PSG: Polysomnography; ECG: Electrocardiogram; AHI: Apnea-Hypopnea Index; SO2: Oxygen Saturation; AHI: Apnea-Hypopnea Index; SPAS: Superior upper airway; MAS: Medium Airway Space; IAS: Inferior Airway Space; PL: Palatal Length; PH: Palatal Height; PA: Palatal Angle; SNML: Mandibular Divergence to Cranial Base; HMI: Hyoid to Mandibular Line; ANB: Maxillo-mandibular angle}

\section{Introduction}

The OSAs (Obstructive Sleep Apnea syndrome) is a condition characterized by repetitive episodes of complete, apnea, or partial, hypopnea, upper airway obstruction occurring during sleep [1]. Its pathogenesis is still unclear but OSAs is divided in two categories: central or obstructive. The central OSAs is related to a lack function of neurological system; the obstructive form is related to a reduction or collapse of upper airway $[2,3]$.

Untreated OSAs is often associated with daytime sleepiness, impaired quality of life and social life [4], many cardiovascular disease as hypertension [5], stroke, heart failure, arterial fibrillation [6,7] and motor vehicle accidents [8]. OSAs affected $25 \%$ of adults [9], $2-7 \%$ of men and $2-5 \%$ in woman [10].

Anamnesis and questionnaires as ESS (Epworth Sleepiness Scale) and Stop Bang model (Stop-Bang Questionnaire) provide measures of the risk of OSAs [11,12]. The gold standard in the OSAs diagnosis is the polysomnography [13]. In obstructive sleep apnea Lateral Headfilms and 3D headfilm are used to evaluate the site of airflow reduction $[14,15]$. These provide to give information about the facial morphology and airflow spaces $[16,17]$.

The study of craniofacial morphology to evaluate the risk of insurgence of OSAs in patients was studied [18]. The Neck circumference, Facial profile, mandible length and mandible position were the most commonly evaluated [19-21].

In the present paper authors evaluated, on supine lateral headfilms, the correlation of UAS (Upper Airway Space), posterior maxillary structures (i.e., palatal morphology), maxillo-mandibular and hyoid position with different severity of OSAs evaluated on polysomnography test.

\section{Material and Methods}

One hundred patients (mean age 51.4 years old; 92 men and 8 women) with OSAs were enrolled in the pre-sent study. The participants and parents provided written informed consent to be 
Citation: Ciavarella D, Laurenziello M, Russo LL, Vocale M, Tepedino M, et al. (2018) Correlation between Craniofacial Structures and Severity of Obstructive Sleep Apnea. Dentistry 8: 525. doi:10.4172/2161-1122.1000525

Page 2 of 7

involved in the study. All patients were visited in the Otorhinolaryngology section at University of Foggia.

\section{Ethical consideration}

This study was based on a retrospective analysis. All patients have signed a specific informed con-sent, which allowed us to analyze the polysomnographic and radiographic data. These data were used for clinical use, and only subsequently they were subject to the statistical analysis.

\section{Patients selection}

Selection of patients with Epworth questionnaire was done. Inclusion criteria were: Epworth scale upper than 10, no smokers, no BMI (body mass index) upper than $34 \mathrm{~kg} / \mathrm{m}^{2}$, no previous maxillofacial and upper airway surgical treatment, no fixed oral appliance or mobile prosthetic rehabilitation, no CPAP (Continuous Positive Airway Pressure) previous treatment, no temporo-mandibular joint disease. Exclusion criteria were: Epworth scale lower than 10, smokers, BMI upper than $34 \mathrm{Kg} / \mathrm{m}^{2}$, previous maxillofacial and upper airway surgical treatment, fixed oral appliance or mobile prosthetic rehabilitation, patients in treatment with CPAP or nCPAP, temporomandibular joint disease.

\section{Instrumental evaluation}

On each patient the head film was done (Gendex GXDP-700). Head films were taken with the patient in supine position fixed in a cephalostat, in centric occlusion, with adequate visualization of reference structures. Landmarks are shown in Figure 1, and described in Table 1. To reduce the error of the method, cephalometric radiographs were selected randomly and reanalyzed 30 days later by the same examiner (M.L. and M.T.).

Each patient received a complete overnight PSG (Polysomnography). All subjects were evaluated for one night in a Sleep Laboratory using a portable device, the Embletta system (Flaga, Reykjavik, Iceland). Recording was performed after one night of adaptation to the hospital setting. Airflow was monitored by a nasal cannula and by oral thermistor. The thoracic-abdominal movements of all subjects were detected through two piezoelectric belts. Overnight continuous recordings of oxy-gen saturation were obtained by finger pulse oximetry. Snoring was recorded by a microphone placed at the neck, and note was taken of ECG (Electrocardiogram) findings and sleep position.

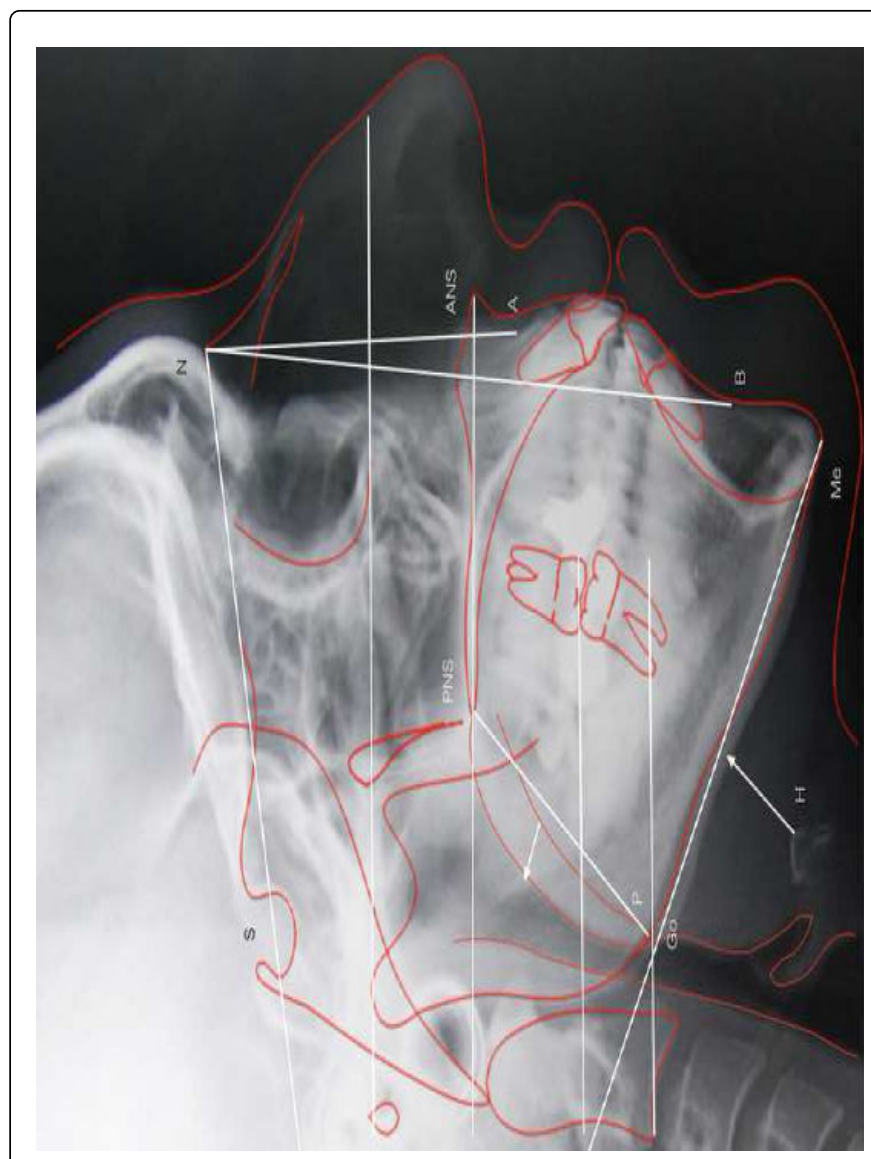

Figure 1: FTIR spectra of silanized SiC fibers $\left(a=1087 \mathrm{~cm}^{-1}, b=815\right.$ $\left.\mathrm{cm}^{-1}\right)$.

\begin{tabular}{|c|l|}
\hline Cephalometrics Landmarks & \\
\hline SPAS & Superior posterior airway space (width of airway behind soft palate along a line parallel to Frankfurt plane) \\
\hline MAS & Medium airway width measured on a line parallel to Frankfurt plane passing through posterior cusps of upper first molar \\
\hline IAS & Inferior airway: width of airway behind tongue along a line parallel to Frankfurt plane passing through Gonion) \\
\hline Palatal Lenght (PL) & Linear distance between Posterior Nasal Spine and the end of soft palate \\
\hline Palatal Angle (PA) & Angle between the Anterior Nasal Spine (ANS)/posterior Nasal Spine (PNS) line and the final part of the Soft Palate (P) \\
\hline Palatal Thickness (PT) & Linear Measurements from the lingual part to the upper airway part \\
\hline ANB & Angle between the NA and NB lines, obtained by subtracting SNB from SNA \\
\hline ML-H & Linear Distance between Mandibular Line and upper Hyoid bone part. \\
\hline SN-ML & Angle between the sella-nasion (SN) line and the mandibular plane (MP). \\
\hline
\end{tabular}

Table 1: Cephalometric landmarks. 
Citation: Ciavarella D, Laurenziello M, Russo LL, Vocale M, Tepedino M, et al. (2018) Correlation between Craniofacial Structures and Severity of Obstructive Sleep Apnea. Dentistry 8: 525. doi:10.4172/2161-1122.1000525

Page 3 of 7

\section{UAS and skeletal structures evaluated}

Authors evaluated and paired the data of PSG with hyoidmandibular position and UAS as follow:

- PSG: SO2 (oxygen saturation), AHI (Apnea-Hypopnea index) and Nadir (i.e., the lowerest oxygen saturation in sleep);

- Respiratory way: SPAS (Superior upper airway); MAS (Medium Airway Space) and IAS (Inferior airway space);

- $\quad$ Maxillary back structures: PL (Palatal Length), PH (Palatal Height), PA (Palatal Angle);

- Hyoid-mandibular structures: SNMI (Mandibular divergence to cranial base), distance of HMI (hyoid to mandibular line);

- ANB (Maxillo-mandibular angle).

\section{Statistical analysis}

Data have been analyzed using GraphPad Prism software 6.0 (GraphPad Prism Software, SanDiego, CA, USA). Presence of normal distribution was assessed by Kolmogorov- Smirnov test and probability plot graph. To evaluate the correlation between PSG data to Maxillomandibular-Hyoid data a non-parametric correlation (Spearman correlation coefficient) was done. Statistical significance was set at 0.05 .

\section{Result}

The results are described in Table 2 and 3 . In table 2 are described the common PSG, craniofacial and UAS data. In table 3 the correlation between the PSG data to the craniofacial structures.

\begin{tabular}{|l|l|l|l|l|l|l|l|l|l|l|l|l|l|}
\hline & AHI & BMI & SO2 & Nadir & Spas & Mas & IAS & ML-H & ANB & SN-ML & PA & PL & PT \\
\hline Mean & 36.28 & 29.24 & 93.28 & 78.8 & 11.82 & 5.92 & 8.909 & 23.21 & 3.832 & 33.15 & 121.1 & 43.27 & 9.78 \\
\hline Std. Deviation & 17.96 & 4.542 & 2.543 & 6.769 & 2.173 & 1.417 & 3.398 & 7.514 & 2.012 & 8.005 & 7.014 & 6.191 & 1.912 \\
\hline Std. Error of Mean & 1.796 & 0.4542 & 0.2543 & 0.6769 & 0.2173 & 0.1417 & 0.3398 & 0.7514 & 0,2012 & 0,8005 & 0,7014 & 0,6191 & 0,1912 \\
\hline Lower 95\% Cl of mean & 32.72 & 28.33 & 92.78 & 77.46 & 11.39 & 5.638 & 8.235 & 21.72 & 3.433 & 31.57 & 119.8 & 42.04 & 9.401 \\
\hline Upper 95\% Cl of mean & 39.85 & 30.14 & 93.78 & 80.14 & 12.25 & 6.201 & 9.583 & 24.7 & 4.231 & 34.74 & 122.5 & 44.5 & 10.16 \\
\hline $\begin{array}{l}\text { Kolmogorov-Smirnoff } \\
\text { test }\end{array}$ & No & No & No & No & Yes & No & Yes & Yes & No & No & No & No & Yes \\
\hline
\end{tabular}

Table 2: Mean and general evaluation of patient's test.

\begin{tabular}{|c|c|c|c|c|c|c|c|c|c|c|c|c|c|}
\hline \multicolumn{2}{|c|}{ Parameters } & \multirow[t]{2}{*}{ AHI } & \multirow{2}{*}{$\begin{array}{c}\text { SO2 } \\
-0.34822\end{array}$} & \multirow{2}{*}{$\begin{array}{c}\text { Nadir } \\
-0.39371\end{array}$} & \multirow{2}{*}{$\begin{array}{c}\text { Spas } \\
-0.12169\end{array}$} & \multirow{2}{*}{$\begin{array}{c}\text { Mas } \\
0.090093\end{array}$} & \multirow{2}{*}{$\begin{array}{c}\text { IAS } \\
0.304711\end{array}$} & \multirow{2}{*}{$\begin{array}{c}\text { H-ML } \\
0.191222\end{array}$} & \multirow{2}{*}{$\begin{array}{c}\text { ANB } \\
0.033843\end{array}$} & \multirow{2}{*}{$\begin{array}{c}\text { SNML } \\
0.106655\end{array}$} & \multirow{2}{*}{$\begin{array}{c}\text { P A } \\
0.051144\end{array}$} & \multirow{2}{*}{$\begin{array}{c}\mathbf{P ~ L} \\
0.075106\end{array}$} & \multirow{2}{*}{$\begin{array}{c}\mathbf{P ~ H} \\
0.192013\end{array}$} \\
\hline & $r$ & & & & & & & & & & & & \\
\hline AHI & $p$ & & 0.000385 & 0.00005 & 0.227785 & 0.372707 & 0.002053 & 0.056676 & 0.738176 & 0.290899 & 0.613318 & 0.457685 & 0.055641 \\
\hline \multirow[b]{2}{*}{ SO2 } & $r$ & -0.348220418 & & 0.319284 & 0.046249 & -0.10673 & -0.4089 & -0.06726 & 0.015669 & 0.107876 & -0.0206 & -0.23898 & -0.1146 \\
\hline & $\mathrm{P}$ & 0.000384915 & & 0.001204 & 0.647731 & 0.290546 & $p<0.001$ & 0.506087 & 0.877033 & 0.285376 & 0.838788 & 0.016641 & 0.256218 \\
\hline \multirow[b]{2}{*}{ Nadir } & $r$ & -0.393709965 & 0.319284 & & -0.04265 & -0.01264 & -0.33434 & -0.04197 & 0.020116 & 0.016355 & -0.21698 & 0.08192 & -0.25171 \\
\hline & $p$ & 5.06644E-05 & 0.001204 & & 0.673547 & 0.900708 & 0.000675 & 0.678426 & 0.842535 & 0.871695 & 0.030125 & 0.41779 & 0.011528 \\
\hline \multirow[b]{2}{*}{ Spas } & $r$ & -0.121689437 & 0.046249 & -0.04265 & & 0.33467 & 0.115879 & 0.061274 & 0.116885 & -0.05162 & 0.007443 & 0.206392 & 0.073192 \\
\hline & $\mathrm{P}$ & 0.227785449 & 0.647731 & 0.673547 & & 0.000666 & 0.250931 & 0.544777 & 0.246811 & 0.610018 & 0.941409 & 0.039379 & 0.469258 \\
\hline \multirow[b]{2}{*}{ Mas } & $r$ & 0.090092686 & -0.10673 & -0.01264 & 0.33467 & & 0.268981 & -0.13797 & 0.08665 & -0.14479 & 0.171897 & 0.31771 & -0.19122 \\
\hline & $\mathrm{P}$ & 0.372706827 & 0.290546 & 0.900707 & 0.000666 & & 0.006809 & 0.17104 & 0.391321 & 0.150628 & 0.087245 & 0.001277 & 0.056677 \\
\hline \multirow[b]{2}{*}{ IAS } & $r$ & 0.304711137 & -0.4089 & -0.33433 & 0.115879 & 0.268981 & & -0.05806 & -0.24281 & -0.32366 & 0.016232 & 0.408471 & 0.33864 \\
\hline & $\mathrm{P}$ & 0.002053009 & $p<0.001$ & 0.000674 & 0.250931 & 0.006809 & & 0.566127 & 0.014927 & 0.00102 & 0.872655 & $p<0.001$ & 0.000568 \\
\hline \multirow[b]{2}{*}{ H-ML } & $r$ & 0.191221668 & -0.06726 & -0.04197 & 0.061274 & -0.13797 & -0.05806 & & 0.403115 & 0.277317 & 0.10358 & 0.171188 & 0.238204 \\
\hline & $\mathrm{P}$ & 0.056675977 & 0.506087 & 0.678425 & 0.544777 & 0.17104 & 0.566127 & & $p<0.001$ & 0.005217 & 0.305109 & 0.088576 & 0.017007 \\
\hline \multirow[b]{2}{*}{ ANB } & $r$ & 0.033842917 & 0.015669 & 0.020116 & 0.116885 & 0.08665 & -0.24281 & 0.403115 & & 0.267579 & -0.18659 & 0.045426 & 0.061128 \\
\hline & $\mathrm{p}$ & 0.738175785 & 0.877033 & 0.842534 & 0.246811 & 0.391321 & 0.014927 & $p<0.001$ & & 0.007115 & 0.063058 & 0.653589 & 0.545735 \\
\hline SNML & $r$ & 0.106654528 & 0.107876 & 0.016355 & -0.05162 & -0.14479 & -0.32366 & 0.277317 & 0.267579 & & 0.064116 & -0.2765 & -0.04408 \\
\hline
\end{tabular}


Citation: Ciavarella D, Laurenziello M, Russo LL, Vocale M, Tepedino M, et al. (2018) Correlation between Craniofacial Structures and Severity of Obstructive Sleep Apnea. Dentistry 8: 525. doi:10.4172/2161-1122.1000525

Page 4 of 7

\begin{tabular}{|c|c|c|c|c|c|c|c|c|c|c|c|c|c|}
\hline & $\mathrm{P}$ & 0.290898735 & 0.285376 & 0.871694 & 0.610018 & 0.150628 & 0.00102 & 0.005217 & 0.007115 & & 0.526242 & 0.005357 & 0.663235 \\
\hline \multirow[b]{2}{*}{ PA } & $r$ & 0.05114414 & -0.0206 & -0.21698 & 0.007443 & 0.171897 & 0.016232 & 0.10358 & -0.18659 & 0.064116 & & 0.015318 & -0.11603 \\
\hline & $\mathrm{p}$ & 0.613318484 & 0.838788 & 0.030125 & 0.941409 & 0.087245 & 0.872655 & 0.305109 & 0.063058 & 0.526242 & & 0.879764 & 0.250304 \\
\hline \multirow[b]{2}{*}{ P L } & r & 0.075105647 & -0.23898 & 0.08191 & 0.206392 & 0.31771 & 0.408471 & 0.171188 & 0.045426 & -0.2765 & 0.015318 & & 0.394957 \\
\hline & $\mathrm{p}$ & 0.457685335 & 0.016641 & 0.41779 & 0.039379 & 0.001277 & $p<0.001$ & 0.088576 & 0.653589 & 0.005357 & 0.879764 & & $p<0.001$ \\
\hline \multirow[b]{2}{*}{ PH } & r & 0.192013064 & -0.1146 & -0.25171 & 0.073192 & -0.19122 & 0.33864 & 0.238204 & 0.061128 & -0.04408 & -0.11603 & 0.394957 & \\
\hline & $\mathrm{p}$ & 0.055640775 & 0.256218 & 0.011528 & 0.469258 & 0.056677 & 0.000568 & 0.017007 & 0.545735 & 0.663235 & 0.250304 & $p<0.001$ & \\
\hline
\end{tabular}

Table 3: Spearman test results.

\section{Kolmogorov-Smirnov test}

Spas, IAS, ML-H and PT passed the test while AHI, BMI, SO2, Nadir, MAS, ANB, SN-ML, PA and PL did not pass the test.

\section{OSA severity- UAS (Table 4)}

Spas presented no relation with OSA severity $(\mathrm{p}=\mathrm{ns})$. MAS had no relation with OSA severity $(\mathrm{p}=\mathrm{ns})$ but presented a relation with IAS ( $\mathrm{r}$ :
$0.2191, \mathrm{p}<0.05)$. IAS presented a great relation with PSG data. IAS had an inverse relation with SO2 ( $\mathrm{r}$ : $-0.4126, \mathrm{p}<0.001)$ and Nadir $(\mathrm{r}$ : $-0.2169, \mathrm{p}<0.05)$; a direct relation between IAS and AHI was shown ( $\mathrm{r}$ : $0.2249, \mathrm{p}<0.001)$.

\begin{tabular}{|l|l|l|l|l|l|l|l|}
\hline Parameters & & AHI & SO2 & Nadir & Spas & Mas & IAS \\
\hline \multirow{4}{*}{ AHI } & $\mathrm{r}$ & & -0.34822 & -0.39371 & -0.12169 & 0.090093 & 0.304711 \\
\cline { 2 - 8 } & $\mathrm{p}$ & & 0.000385 & 0.00005 & 0.227785 & 0.372707 & 0.002053 \\
\hline \multirow{3}{*}{ SO2 } & $\mathrm{r}$ & -0.34822 & & 0.319284 & 0.046249 & -0.10673 & -0.4089 \\
\cline { 2 - 9 } & $\mathrm{p}$ & 0.000385 & & 0.001204 & 0.647731 & 0.290546 & $0<0.001$ \\
\hline \multirow{3}{*}{ Spas } & $\mathrm{r}$ & -0.39371 & 0.319284 & & -0.04265 & -0.01264 & -0.33434 \\
\hline \multirow{3}{*}{ Mas } & $\mathrm{p}$ & $5.07 \mathrm{E}-05$ & 0.001204 & & 0.673547 & 0.900708 & 0.000675 \\
\hline \multirow{3}{*}{ IAS } & $\mathrm{r}$ & -0.12169 & 0.046249 & -0.04265 & & 0.33467 & 0.115879 \\
\cline { 2 - 9 } & $\mathrm{p}$ & 0.227785 & 0.647731 & 0.673547 & & 0.000666 & 0.250931 \\
\hline
\end{tabular}

Table 4: Spearman correlation for polysomnography and upper air way.

Maxillo-mandibular-hyoid position and OSA severity (Table 5)

No statistical relation with OSA severity was shown.

\begin{tabular}{|c|c|c|c|c|c|c|c|}
\hline \multicolumn{2}{|c|}{$\begin{array}{l}\text { Parameter } \\
\text { s }\end{array}$} & \multirow[t]{2}{*}{$\mathrm{AHI}$} & \multirow{2}{*}{$\begin{array}{l}\text { SO2 } \\
-0.34822\end{array}$} & \multirow{2}{*}{$\begin{array}{l}\text { Nadir } \\
-0.39371\end{array}$} & \multirow{2}{*}{$\begin{array}{l}\text { H-ML } \\
\begin{array}{l}0.19122 \\
2\end{array}\end{array}$} & \multirow{2}{*}{$\begin{array}{l}\text { ANB } \\
0.03384 \\
3\end{array}$} & \multirow{2}{*}{$\begin{array}{l}\text { SNML } \\
0.10665 \\
5\end{array}$} \\
\hline & $r$ & & & & & & \\
\hline $\mathrm{AHI}$ & $p$ & & $\begin{array}{l}0.00038 \\
5\end{array}$ & 0.00005 & $\begin{array}{l}0.05667 \\
6\end{array}$ & $\begin{array}{l}0.73817 \\
6\end{array}$ & $\begin{array}{l}0.29089 \\
9\end{array}$ \\
\hline
\end{tabular}

\begin{tabular}{|c|c|c|c|c|c|c|c|}
\hline & $r$ & -0.34822 & & $\begin{array}{l}0.31928 \\
4\end{array}$ & -0.06726 & $\begin{array}{l}0.01566 \\
9\end{array}$ & $\begin{array}{l}0.10787 \\
6\end{array}$ \\
\hline SO2 & $\mathrm{P}$ & $\begin{array}{l}0.00038 \\
5\end{array}$ & & $\begin{array}{l}0.00120 \\
4\end{array}$ & $\begin{array}{l}0.50608 \\
7\end{array}$ & $\begin{array}{l}0.87703 \\
3\end{array}$ & $\begin{array}{l}0.28537 \\
6\end{array}$ \\
\hline & $r$ & -0.39371 & $\begin{array}{l}0.31928 \\
4\end{array}$ & & -0.04197 & 0.020116 & $\begin{array}{l}0.01635 \\
5\end{array}$ \\
\hline Nadir & $\mathrm{p}$ & $5.07 \mathrm{E}-05$ & $\begin{array}{l}0.00120 \\
4\end{array}$ & & $\begin{array}{l}0.67842 \\
6\end{array}$ & $\begin{array}{l}0.84253 \\
5\end{array}$ & $\begin{array}{l}0.87169 \\
5\end{array}$ \\
\hline H-ML & $r$ & $\begin{array}{l}0.19122 \\
2\end{array}$ & -0.06726 & -0.04197 & & 0.403115 & $\begin{array}{l}0.27731 \\
7\end{array}$ \\
\hline
\end{tabular}


Citation: Ciavarella D, Laurenziello M, Russo LL, Vocale M, Tepedino M, et al. (2018) Correlation between Craniofacial Structures and Severity of Obstructive Sleep Apnea. Dentistry 8: 525. doi:10.4172/2161-1122.1000525

Page 5 of 7

\begin{tabular}{|c|c|c|c|c|c|c|c|}
\hline & $\mathrm{p}$ & $\begin{array}{l}0.05667 \\
6\end{array}$ & $\begin{array}{l}0.50608 \\
7\end{array}$ & $\begin{array}{l}0.67842 \\
5\end{array}$ & & $p<0.001$ & $\begin{array}{l}0.00521 \\
7\end{array}$ \\
\hline \multirow[b]{2}{*}{ ANB } & $r$ & $\begin{array}{l}0.03384 \\
3\end{array}$ & $\begin{array}{l}0.01566 \\
9\end{array}$ & 0.020116 & 0.403115 & & $\begin{array}{l}0.26757 \\
9\end{array}$ \\
\hline & $p$ & $\begin{array}{l}0.73817 \\
6\end{array}$ & $\begin{array}{l}0.87703 \\
3\end{array}$ & $\begin{array}{l}0.84253 \\
4\end{array}$ & $p<0.001$ & & 0.007115 \\
\hline \multirow[b]{2}{*}{ SNML } & $r$ & $\begin{array}{l}0.10665 \\
5\end{array}$ & $\begin{array}{l}0.10787 \\
6\end{array}$ & $\begin{array}{l}0.01635 \\
5\end{array}$ & $\begin{array}{l}0.27731 \\
7\end{array}$ & $\begin{array}{l}0.26757 \\
9\end{array}$ & \\
\hline & $p$ & $\begin{array}{l}0.29089 \\
9\end{array}$ & $\begin{array}{l}0.28537 \\
6\end{array}$ & $\begin{array}{l}0.87169 \\
4\end{array}$ & $\begin{array}{l}0.00521 \\
7\end{array}$ & 0.007115 & \\
\hline
\end{tabular}

Table 5: Spearman correlation for polysonmnography and mandibular hyoid.

\section{Palatal structure and OSA severity (Table 6)}

PH had no statistical relation with AHI and $\mathrm{SO} 2(\mathrm{p}=\mathrm{ns})$, but had and inverse correlation with Nadir ( $\mathrm{r}:-0.21698$; $\mathrm{p}<0.05)$. PA presented an inverse relation with Nadir $(\mathrm{r}:-0.169, \mathrm{p}<0.05)$ and a relation with MAS ( $r: 0.2375, p<0.05)$. An inverse relation between the PL and SO2 $(\mathrm{r}:-0.2324, \mathrm{p}<0.05)$ and direct relation with IAS $(\mathrm{r}: 0.2765, \mathrm{p}<0.05)$ was shown.

\section{UAS and maxillo-mandibular hyoid position}

SNML had an inverse relation with IAS $(\mathrm{r}:-0.32, \mathrm{p}<0.05)$. ANB had an inverse relation to IAS ( $\mathrm{r}:-0.24, \mathrm{p}<0.05)$; No correlation between the hyoid position and the upper airway space was found (Table 7).

\begin{tabular}{|c|c|c|c|c|c|c|c|}
\hline \multicolumn{2}{|c|}{$\begin{array}{l}\text { Parameter } \\
\text { s }\end{array}$} & \multirow[t]{2}{*}{ AHI } & \multirow{2}{*}{$\begin{array}{l}\text { SO2 } \\
-0.34822\end{array}$} & \multirow{2}{*}{$\begin{array}{l}\text { Nadir } \\
-0.39371\end{array}$} & \multirow{2}{*}{$\begin{array}{l}\text { PA } \\
0.051144\end{array}$} & \multirow{2}{*}{$\begin{array}{l}\text { PL } \\
\begin{array}{l}0.07510 \\
6\end{array}\end{array}$} & \multirow{2}{*}{$\begin{array}{l}\text { PT } \\
0.19201 \\
3\end{array}$} \\
\hline & $r$ & & & & & & \\
\hline AHI & $\mathrm{p}$ & & $\begin{array}{l}0.00038 \\
5\end{array}$ & 0.00005 & $\begin{array}{l}0.61331 \\
8\end{array}$ & $\begin{array}{l}0.45768 \\
5\end{array}$ & $\begin{array}{l}0.05564 \\
1\end{array}$ \\
\hline \multirow[b]{2}{*}{ SO2 } & $r$ & -0.34822 & & $\begin{array}{l}0.31928 \\
4\end{array}$ & -0.0206 & -0.23898 & -0.1146 \\
\hline & $P$ & $\begin{array}{l}0.00038 \\
5\end{array}$ & & $\begin{array}{l}0.00120 \\
4\end{array}$ & $\begin{array}{l}0.83878 \\
8\end{array}$ & $\begin{array}{l}0.01664 \\
1\end{array}$ & $\begin{array}{l}0.25621 \\
8\end{array}$ \\
\hline \multirow[b]{2}{*}{ Nadir } & $r$ & -0.39371 & $\begin{array}{l}0.31928 \\
4\end{array}$ & & -0.21698 & 0.08192 & -0.25171 \\
\hline & $\mathrm{p}$ & 5.07E-05 & $\begin{array}{l}0.00120 \\
4\end{array}$ & & $\begin{array}{l}0.03012 \\
5\end{array}$ & 0.41779 & 0.011528 \\
\hline \multirow[b]{2}{*}{ PA } & $r$ & 0.051144 & -0.0206 & -0.21698 & & $\begin{array}{l}0.01531 \\
8\end{array}$ & -0.11603 \\
\hline & $\mathrm{p}$ & $\begin{array}{l}0.61331 \\
8\end{array}$ & $\begin{array}{l}0.83878 \\
8\end{array}$ & $P<0.05$ & & $\begin{array}{l}0.87976 \\
4\end{array}$ & $\begin{array}{l}0.25030 \\
4\end{array}$ \\
\hline \multirow[b]{2}{*}{ PL } & $r$ & $\begin{array}{l}0.07510 \\
6\end{array}$ & -0.23898 & 0.08191 & $\begin{array}{l}0.01531 \\
8\end{array}$ & & $\begin{array}{l}0.39495 \\
7\end{array}$ \\
\hline & $\mathrm{p}$ & $\begin{array}{l}0.45768 \\
5\end{array}$ & $\begin{array}{l}0.01664 \\
1\end{array}$ & 0.41779 & $\begin{array}{l}0.87976 \\
4\end{array}$ & & $p<0.001$ \\
\hline PT & $r$ & $\begin{array}{l}0.19201 \\
3\end{array}$ & -0.1146 & -0.25171 & -0.11603 & $\begin{array}{l}0.39495 \\
7\end{array}$ & \\
\hline
\end{tabular}

\begin{tabular}{|c|c|c|c|c|c|}
\hline$p$ & $\begin{array}{l}0.05564 \\
1\end{array}$ & $\begin{array}{l}0.25621 \\
8\end{array}$ & 0.011528 & $\begin{array}{l}0.25030 \\
4\end{array}$ & $p<0.001$ \\
\hline
\end{tabular}

Table 6: Spearman correlation for polysomnography and palatal structure.

\begin{tabular}{|c|c|c|c|c|c|c|c|}
\hline \multicolumn{2}{|c|}{$\begin{array}{l}\text { Parameter } \\
\text { s }\end{array}$} & \multirow[t]{2}{*}{ Spas } & \multirow{2}{*}{$\begin{array}{l}\text { Mas } \\
0.33467\end{array}$} & \multirow{2}{*}{$\begin{array}{l}\text { IAS } \\
0.115879\end{array}$} & \multirow{2}{*}{$\begin{array}{l}\text { H-ML } \\
0.06127 \\
4\end{array}$} & \multirow{2}{*}{$\begin{array}{l}\text { ANB } \\
0.116885\end{array}$} & \multirow{2}{*}{$\begin{array}{l}\text { SNML } \\
-0.05162\end{array}$} \\
\hline & $r$ & & & & & & \\
\hline Spas & $\mathrm{P}$ & & $\begin{array}{l}0.00066 \\
6\end{array}$ & $\begin{array}{l}0.25093 \\
1\end{array}$ & $\begin{array}{l}0.54477 \\
7\end{array}$ & 0.246811 & $\begin{array}{l}0.61001 \\
8\end{array}$ \\
\hline \multirow[b]{2}{*}{ Mas } & $r$ & 0.33467 & & $\begin{array}{l}0.26898 \\
1\end{array}$ & -0.13797 & 0.08665 & -0.14479 \\
\hline & $\mathrm{P}$ & $\begin{array}{l}0.00066 \\
6\end{array}$ & & $\begin{array}{l}0.00680 \\
9\end{array}$ & 0.17104 & $\begin{array}{l}0.39132 \\
1\end{array}$ & $\begin{array}{l}0.15062 \\
8\end{array}$ \\
\hline \multirow[b]{2}{*}{ IAS } & $r$ & 0.115879 & $\begin{array}{l}0.26898 \\
1\end{array}$ & & -0.05806 & -0.24281 & -0.32366 \\
\hline & $\mathrm{P}$ & $\begin{array}{l}0.25093 \\
1\end{array}$ & $\begin{array}{l}0.00680 \\
9\end{array}$ & & $\begin{array}{l}0.56612 \\
7\end{array}$ & $\begin{array}{l}0.01492 \\
7\end{array}$ & 0.00102 \\
\hline \multirow[b]{2}{*}{ H-ML } & $r$ & $\begin{array}{l}0.06127 \\
4\end{array}$ & -0.13797 & -0.05806 & & 0.403115 & $\begin{array}{l}0.27731 \\
7\end{array}$ \\
\hline & $\mathrm{P}$ & $\begin{array}{l}0.54477 \\
7\end{array}$ & 0.17104 & $\begin{array}{l}0.56612 \\
7\end{array}$ & & $p<0.001$ & $\begin{array}{l}0.00521 \\
7\end{array}$ \\
\hline \multirow[b]{2}{*}{ ANB } & $r$ & 0.116885 & 0.08665 & -0.24281 & 0.403115 & & $\begin{array}{l}0.26757 \\
9\end{array}$ \\
\hline & $\mathrm{p}$ & 0.246811 & $\begin{array}{l}0.39132 \\
1\end{array}$ & $\begin{array}{l}0.01492 \\
7\end{array}$ & $p<0.001$ & & 0.007115 \\
\hline \multirow[b]{2}{*}{ SNML } & $r$ & -0.05162 & -0.14479 & -0.32366 & $\begin{array}{l}0.27731 \\
7\end{array}$ & $\begin{array}{l}0.26757 \\
9\end{array}$ & \\
\hline & $\mathrm{P}$ & $\begin{array}{l}0.61001 \\
8\end{array}$ & $\begin{array}{l}0.15062 \\
8\end{array}$ & 0.00102 & $\begin{array}{l}0.00521 \\
7\end{array}$ & 0.007115 & \\
\hline
\end{tabular}

Table 7: Spearman correlation for upper air way and mandibular hyoid.

\section{UAS and palatal structures}

PA had non-relation with UAS; PL presented a correlation with Spas ( $\mathrm{r}: 0.20 ; \mathrm{P}<0.05)$, Mas ( $\mathrm{r}: 0.31 ; \mathrm{P}<0.05)$ and $(\mathrm{r}: 0.40 ; \mathrm{P}<0.001)$; $\mathrm{PT}$ presented a correlation with IAS $(\mathrm{r}: 0.33 ; \mathrm{P}<0.001)$ (Table 8$)$.

\begin{tabular}{|c|c|c|c|c|c|c|c|}
\hline \multicolumn{2}{|c|}{$\begin{array}{l}\text { Parameter } \\
\text { s }\end{array}$} & Spas & Mas & IAS & PA & PL & PT \\
\hline \multirow[b]{2}{*}{$\begin{array}{l}\text { Spa } \\
\text { s }\end{array}$} & $r$ & & 0.33467 & 0.115879 & $\begin{array}{l}0.00744 \\
3\end{array}$ & $\begin{array}{l}0.20639 \\
2\end{array}$ & $\begin{array}{l}0.07319 \\
2\end{array}$ \\
\hline & $\mathrm{p}$ & & $\begin{array}{l}0.00066 \\
6\end{array}$ & $\begin{array}{l}0.25093 \\
1\end{array}$ & $\begin{array}{l}0.94140 \\
9\end{array}$ & $\begin{array}{l}0.03937 \\
9\end{array}$ & $\begin{array}{l}0.46925 \\
8\end{array}$ \\
\hline \multirow[b]{2}{*}{ Mas } & $r$ & 0.33467 & & $\begin{array}{l}0.26898 \\
1\end{array}$ & $\begin{array}{l}0.17189 \\
7\end{array}$ & 0.31771 & -0.19122 \\
\hline & $\mathrm{p}$ & $\begin{array}{l}0.00066 \\
6\end{array}$ & & $\begin{array}{l}0.00680 \\
9\end{array}$ & $\begin{array}{l}0.08724 \\
5\end{array}$ & $\begin{array}{l}0.00127 \\
7\end{array}$ & $\begin{array}{l}0.05667 \\
7\end{array}$ \\
\hline IAS & $r$ & 0.115879 & $\begin{array}{l}0.26898 \\
1\end{array}$ & & $\begin{array}{l}0.01623 \\
2\end{array}$ & $\begin{array}{l}0.40847 \\
1\end{array}$ & 0.33864 \\
\hline
\end{tabular}




\begin{tabular}{|c|c|c|c|c|c|c|c|}
\hline & $p$ & $\begin{array}{l}0.25093 \\
1\end{array}$ & $\begin{array}{l}0.00680 \\
9\end{array}$ & & $\begin{array}{l}0.87265 \\
5\end{array}$ & $p<0.001$ & $\begin{array}{l}0.00056 \\
8\end{array}$ \\
\hline \multirow[b]{2}{*}{ PA } & $r$ & $\begin{array}{l}0.00744 \\
3\end{array}$ & $\begin{array}{l}0.17189 \\
7\end{array}$ & $\begin{array}{l}0.01623 \\
2\end{array}$ & & $\begin{array}{l}0.01531 \\
8\end{array}$ & -0.11603 \\
\hline & $p$ & $\begin{array}{l}0.94140 \\
9\end{array}$ & $\begin{array}{l}0.08724 \\
5\end{array}$ & $\begin{array}{l}0.87265 \\
5\end{array}$ & & $\begin{array}{l}0.87976 \\
4\end{array}$ & $\begin{array}{l}0.25030 \\
4\end{array}$ \\
\hline \multirow[b]{2}{*}{ P L } & $r$ & $\begin{array}{l}0.20639 \\
2\end{array}$ & 0.31771 & $\begin{array}{l}0.40847 \\
1\end{array}$ & $\begin{array}{l}0.01531 \\
8\end{array}$ & & $\begin{array}{l}0.39495 \\
7\end{array}$ \\
\hline & $p$ & $\begin{array}{l}0.03937 \\
9\end{array}$ & $\begin{array}{l}0.00127 \\
7\end{array}$ & $p<0.001$ & $\begin{array}{l}0.87976 \\
4\end{array}$ & & $p<0.001$ \\
\hline \multirow[b]{2}{*}{ PT } & $r$ & $\begin{array}{l}0.07319 \\
2\end{array}$ & -0.19122 & 0.33864 & -0.11603 & $\begin{array}{l}0.39495 \\
7\end{array}$ & \\
\hline & $p$ & $\begin{array}{l}0.46925 \\
8\end{array}$ & $\begin{array}{l}0.05667 \\
7\end{array}$ & $\begin{array}{l}0.00056 \\
8\end{array}$ & $\begin{array}{l}0.25030 \\
4\end{array}$ & $p<0.001$ & \\
\hline
\end{tabular}

Table 8: Spearman correlation for upper air way and palatal structures.

\section{Discussion}

OSAs is a common condition that affect child and adults. Clinically two forms of OSA are decrypted: the primary and the secondary form. An alteration of the central nervous system is present in the primary form (central form) in which alteration of ventilation is related to an incoming of the nervous stimulus at the respiratory system. This type of OSAs has a more complex diagnosis and is more difficult to treat [22]. Reduction of upper airways width, obesity, obstructive factors such as adenoide-tonsillae hypertrophy, PAS reduction, redundant soft palate, craniofacial structures and prominent base of tongue were included in the secondary form classification [18].

Both the forms include a reduction of respiratory flow with an increase of bloody pressure and a reduction of oxygen saturation.

The secondary form is related to an obstruction of upper airway. These structures may be influenced by the maxillo-mandibular-hyoid position [23].

In the last years a great correlation of mandibular and hyoid position was shown. Many authors suggest that patients with a backward mandibular position and an increase of distance of hyoid to mandibular pane presented an increase of secondary OSAs risk [24].

The evaluation of cranio-facial structures included the palatal lenght and thickness too. An increase of palatal length and thickness were included as risk factors for secondary OSA pathogenesis [24,25].

In the present paper authors evaluated one-hundred patients affected by a mild to severe OSAs. A supine head-films was done end evaluated by a cephalometric analysis. Authors paired the cephalometric data to PSG data to evaluate the possible correlation between the craniofacial structures and secondary OSA.

The evaluation of cephalometric analysis showed a prevalence of backward and post routed mandible (ANB mean 3.832; SN-ML mean $33.15^{\circ}$ ), and an increase of ML-H (mean $23.21 \mathrm{~mm}$ ). The upper airway space was reduced (SPAs $11.82 \mathrm{~mm}$; Mas $5.92 \mathrm{~mm}$; IAS $8.909 \mathrm{~mm}$ ) and the palatal structure was increased (Length $43.27 \mathrm{~mm}$; height $9.78 \mathrm{~mm}$ and angle $121.1^{\circ}$ ). The present data was similar to other researches [24-28].

In the present study a possible correlation between the cranio-facial structures and UAS was evaluated. The UAS influenced the severity of
OSA in the inferior part (i.e., IAS). IAS had a relation with AHI and inverse relation with S02 and Nadir. Spas and Mas were not statistically related to OSA severity.

The correlation test (i.e., Spearman test) showed how the SPas and Mas had no correlation with the severity of OSA but a linear relation of IAS to AHI was evaluated. An increase of IAS was correlated to an increase of $\mathrm{AHI}$, a reduction of $\mathrm{SO} 2$ and Nadir. Authors showed how IAS had correlation with AHI, SO2 and Nadir. No other structures presented a strict relation with PSG data. The maxillo-mandibular structures had no statistically relation with OSA severity; the palatal structure influenced the Nadir $(\mathrm{PA} p<0.05$; PT $\mathrm{p}<0.05)$ and SO2 (PL $\mathrm{p}<0.05)$.

In the correlation test is showed how IAS was influenced by the mandibular position (i.e., a backward and post inclined position of the jaw increase the IAS). UA was not influenced by the Hyoid distance from the mandibular plane. A great correlation of PL $(\mathrm{p}<0.001)$ and PT $(\mathrm{p}<0.001)$ with IAS was evaluated.

\section{Conclusion}

In the present paper authors evaluated one -hundred patients affected by secondary OSA. The study was done with a supine laterolateral headfilms. The cephalometric evaluation showed how patients presented:

a) Reduction of UAS;

b) Post-inclination of the mandible;

c) Increase of the ANB Angle

d) Increase of palatal length, palatal height and palatal angle.

Authors correlated the craniofacial structures (maxillo-mandibularhyoid position, palatal length, thickness and angle) and the upper airway space (IAS, MAS, sPAS) to the PSG data (AHI, SO2 and Nadir).

The papers showed:

- A great correlation between IAS and OSA data;

- A direct correlation between the palatal length and thickness with IAS;

- An inverse correlation between the mandibular post-inclination and backward position and IAS.

The paper presented three main limitations: 1) low numbers of patients evaluated; 2) BMI and 3) craniofacial structures evaluation on a bidimensional headfilms (i.e., latero-lateral headfilms). The strength of the paper was due to the fact that patients were evaluated in the supine position and the exclusion of excessive smoker patients.

\section{References}

1. Disorders AAoS (2005) International Classification of Sleep Disorders, Second Edition. American Academy of Sleep Medicine. Westchester, IL.

2. Sanchez-de-la-Torre M, Mediano O, Barcelo A, Pierola J, De la Pena M, et al. (2011) The influence of obesity and obstructive sleep apnea on metabolic hormones. Sleep breath 16: 649-656.

3. Shamsuzzaman AS, Gersh BJ, Somers VK (2003) Obstructive sleep apnea: implications for cardiac and vascular disease. JAMA 290: 1906-1914.

4. Johnston CD, Gleadhill IC, Cinnamond MJ, Gabbey J, Burden DJ (2002) Mandibular advancement appliances and obstructive sleep apnoea: a randomized clinical trial. Eur J Orthod 24: 251-262. 
Citation: Ciavarella D, Laurenziello M, Russo LL, Vocale M, Tepedino M, et al. (2018) Correlation between Craniofacial Structures and Severity of Obstructive Sleep Apnea. Dentistry 8: 525. doi:10.4172/2161-1122.1000525

Page 7 of 7

5. Peppard PE, Young T, Palta M, Skatrud J (2000) Prospective study of the association between sleep-disordered breathing and hypertension. $\mathrm{N}$ Engl J Med 342: 1378-1384.

6. Shahar E, Whitney CW, Redline S, et al. (2001) Sleep-disordered breathing and cardiovascular disease: cross-sectional results of the Sleep Heart Health Study. Am J Respir Crit Care Med 163: 19-25.

7. $\mathrm{Ng} \mathrm{A}$, Gotsopoulos H, Darendeliler AM, Cistulli PA (2005) Oral appliance therapy for obstructive sleep apnea. Treat Respir Med 4: 409-422.

8. Haraldsson PO, Carenfelt C, Diderichsen F, Nygren A, Tingvall C (1990) Clinical symptoms of sleep apnea syndrome and automobile accidents. ORL J Otorhinolaryngol Relat Spec 52: 57-62.

9. Bixler EO, Vgontzas AN, Ten Have T, Tyson K, Kales A (1998) Effects of age on sleep apnea in men: I. Prevalence and severity. Am J Respir Crit Care Med 157: 144-148.

10. Young T, Palta M, Dempsey J, Skatrud J, Weber S, et al. (1993) The occurrence of sleep-disordered breathing among middle-aged adults. $\mathrm{N}$ Engl J Med 328: 1230-1235.

11. Johns MW (1991) A new method for measuring daytime sleepiness: the Epworth sleepiness scale. Sleep 14: 540-545.

12. Chung F, Yegneswaran B, Liao P, Chung SA, Vairavanathan S, et al. (2008) STOP questionnaire: a tool to screen patients for obstructive sleep apnea. Anesthesiology 108: 812-821.

13. Steyer BJ, Quan SF, Morgan WJ (1985) Polysomnography scoring for sleep apnea. Use of a sampling method. Am Rev Respir Dis 131: 592-595.

14. Beranger T, Garreau E, Ferri J, Raoul G (2017) Morphological impact on patients of maxillomandibular advancement surgery for the treatment of obstructive sleep apnea-hypopnea syndrome. Int Orthod 15: 40-53.

15. Jayaratne YSN, Elsharkawi I, Macklin EA (2017) The facial morphology in Down syndrome: A 3D comparison of patients with and without obstructive sleep apnea. American journal of medical genetics Part A 173 3013-3021.

16. Sokucu O, Oksayan R, Uyar M, Ademci KE, Usumez S (2016) Relationship between head posture and the severity of obstructive sleep apnea. Am J Orthod Dentofacial Orthop 150: 945-949.

17. Adisen MZ, Misirlioglu M, Yorubulut S, Nalcaci R (2017) Correlation of upper airway radiographic measurements with risk status for obstructive sleep apnea syndrome in young dental patients. Oral Surg Oral Med Oral Pathol Oral Radiol 123: 129-136.

18. Agha B, Johal A (2017) Facial phenotype in obstructive sleep apneahypopnea syndrome: a systematic review and meta-analysis. J Sleep Res 26: 122-131.

19. Perri RA, Kairaitis K, Cistulli P, Wheatley JR, Amis TC (2014) Surface cephalometric and anthropometric variables in OSA patients: statistical models for the OSA phenotype. Sleep Breath 18: 39-52.

20. Lee RW, Chan AS, Grunstein RR, Cistulli PA (2009) Craniofacial phenotyping in obstructive sleep apnea- a novel quantitative photographic approach. Sleep 32: 37-45.

21. Banabilh SM, Samsudin AR, Suzina AH, Dinsuhaimi S (2010) Facial profile shape, malocclusion and palatal morphology in Malay obstructive sleep apnea patients. Angle Orthod 80: 37-42.

22. Ferini-Strambi L, Lombardi GE, Marelli S, Galbiati A (2017) Neurological Deficits in Obstructive Sleep Apnea. Curr Treat Options Neurol 19: 16.

23. Cunha TCA, Guimaraes TM, Schultz TCB, De Almeida FR, Cunha TM, et al. (2017) Predictors of success for mandibular repositioning appliance in obstructive sleep apnea syndrome. Braz Oral Res 31: e37.

24. Bayat M, Shariati M, Rakhshan V, Fateh A, Sobouti F, et al. (2017) Cephalometric risk factors of obstructive sleep apnea. Cranio 35: 321-326.

25. Lim JS, Lee JW, Han C, Kwon JW (2017) Correlation of soft palate length with velum obstruction and severity of obstructive sleep apnea syndrome. Auris Nasus Larynx 45: 499-503.

26. Lee CH, Kim JW, Lee HJ, Yun PY, Kim DY, et al. (2009) An investigation of upper airway changes associated with mandibular advancement device using sleep videofluoroscopy in patients with obstructive sleep apnea. Arch Otolaryngol Head Neck Surg 135: 910-914.

27. Lee CH, Mo JH, Kim BJ, et al. (2009) Evaluation of soft palate changes using sleep videofluoroscopy in patients with obstructive sleep apnea. Arch Otolaryngol Head Neck Surg 135: 168-172.

28. Davoudmanesh Z, Bayat M, Abbasi M, Rakhshan V, Shariati M (2017) Cephalometric risk factors associated with myocardial infarction in patients suffering from obstructive sleep apnea: A pilot case-control study. Cranio 35: 15-18. 